\title{
测定森林树木叶面积的最适叶片数是多少?
}

\author{
高思涵 葛玨希 周李奕 朱宝琳 葛星宇李 凯“倪 健 \\ 浙江师范大学化学与生命科学学院, 浙江金华 321004
}

\begin{abstract}
摘 要 植物形态性状叶面积简单易测, 能够反映植物对环境的适应与响应, 指示生态系统的功能与过程。在野外测定叶面 积时, 叶片取样数量往往采用约定俗成的10-20片, 但到底采集多少叶片才是最优和最具代表性, 却少有探究。该研究以浙江 金华山常绿落叶阔叶混交林的优势树种木荷(Schima superba)与枫香树(Liquidambar formosana)为研究对象, 通过对 5 个胸径 等级植株和每个植株 6 个方位开展大批量叶片取样( $>2500$ 个), 分析两个树种的叶面积变异特征, 探讨叶片取样数量为多少才 能最代表该森林类型的叶片大小性状规律。结果表明, 常绿乔木木荷平均叶面积与变幅均小于落叶乔木枫香树。木荷叶面积 与胸径无显著相关性, 而枫香树叶面积与胸径有较显著相关性, 但两个树种均在中胸径等级 $(15-20 \mathrm{~cm})$ 差异不显著; 两个树 种的叶面积与采样方位无显著相关性, 但在东、西和底部的差异不显著。因此, 综合考虑代表性与野外可操作性, 叶片采集 首选中胸径成树的底部叶片。随机抽样统计可知, 树木叶面积测定的最适叶片采集数量因物种而异, 木荷的最适叶片采集数 量为 40 , 而枫香树最少为 170 片。因此, 在叶面积测定时, 叶片采集的数量应该不能只局限在10-20片, 在人力、物力和时间等 条件允许的情况下, 应该尽可能多地测定较多叶片的叶面积。
\end{abstract}

关键词 植物功能性状; 叶面积; 取样数量; 取样方位; 树龄; 常绿落叶阔叶混交林

高思涵, 葛玨希, 周李奕, 朱宝琳, 葛星宇, 李凯, 倪健 (2018). 测定森林树木叶面积的最适叶片数是多少? 植物生态学报, 42, 917-925. DOI: 10.17521/cjpe.2018.0087

\section{What is the optimal number of leaves when measuring leaf area of tree species in a forest community?}

GAO Si-Han, GE Yu-Xi, ZHOU Li-Yi, ZHU Bao-Lin, GE Xing-Yu, LI Kai*, and NI Jian*

College of Chemistry and Life Sciences, Zhejiang Normal University, Jinhua, Zhejiang 321004, China

\begin{abstract}
Aims Leaf size, as one of the easier measured plant morphological traits, reflects response and adaptation of plants to environment and indicates functions and processes of ecosystem. When measuring leaf size (the leaf area, $L A)$ on the field, the common accepted practice considers that the number of leaves picked off is often 10-20. However, what is the optimal number of leaves remains unknown. In this study, we attempt to determine how many leaves should be investigated when the leaf size of a tree is measured.

Methods This study selected two dominant tree species (Schima superba, Ss and Liquidambar formosana, Lf) from a broadleaved evergreen and deciduous mixed forest in Jinhua Mountain of Zhejiang Province, eastern China. On the basis of sampling (>2 500 leaves for each tree) in five classes of the diameter of breast height ( $D B H$ ) of tree species and at six directions for each individual, variations of $L A$ in the two tree species are statistically analyzed. The optimal number of leaves, which can mostly represent the common leaf size feature, is further investigated.

Important findings Mean LA of the evergreen tree Ss was smaller than that of the deciduous tree Lf. The former was $(41.60 \pm 10.88) \mathrm{cm}^{2}\left(16.74-100.80 \mathrm{~cm}^{2}\right)$ and the latter was $(57.65 \pm 19.35) \mathrm{cm}^{2}\left(11.31-129.51 \mathrm{~cm}^{2}\right) . L A$ of Lf was significantly related to the $D B H$, but $L A$ of Ss was not. $L A$ of both trees in the middle $D B H$ class (15-20 cm) was not significantly different from their means. LA of two trees have no significant correlations with the sampling directions, but $L A$ at the east, west and bottom had no significant relationships with their means. Considering the representativeness and practicality in the field sampling, the priority of selecting leaves can target the bottom direction of middle diameter mature trees. Random sampling analysis indicated that, the optimal number of leaves
\end{abstract}

收稿日期Received: 2018-04-16 接受日期Accepted: 2018-06-09

基金项目: 国家自然科学基金(41471049)。Supported by the National Natural Science Foundation of China (41471049).

* 通信作者Corresponding author (Li K: likai@zjnu.edu.cn; Ni J: nijian@zjnu.edu.cn) 
for tree $L A$ measurement is species specific. The optimal number of leaves for Ss is 40 and for Lf is at least 170 , respectively. Therefore, when measuring leaf area in a forest community, the optimal sampling number of leaves should not be limited to 10-20 leaves. Under sufficient labor, material and time, more leaves should be measured.

Key words plant functional traits; leaf area; sampling number; sampling direction; tree age; evergreen and deciduous broadleaved mixed forest

Gao SH, Ge YX, Zhou LY, Zhu BL, Ge XY, Li K, Ni J (2018). What is the optimal number of leaves when measuring leaf area of tree species in a forest community? Chinese Journal of Plant Ecology, 42, 917-925. DOI: 10.17521/cjpe.2018.0087

叶片是植物光合作用和物质生产的主要器官, 指示叶片大小的性状，如叶面积(叶片单面投影表 面积, $\mathrm{mm}^{2}$ ), 可反映植物对环境的响应与适应, 指 示生态系统的功能与过程, 与环境胁迫、干扰和植 物的生长对策及其利用资源的能力密切相关, 是重 要的植物功能性状之一(Wright et al., 2004; 孟婷婷 等, 2007; 刘晓娟和马克平, 2015; Díaz et al., 2016)。 相对于茎干与根系性状, 叶片易于采样, 性状易于 测定, 可以通过大批量的采样来比较和分析不同地 区、不同生态系统和不同植物种类的功能性状特征。 同时, 叶面积性状还可拓展用以计算比叶面积、基 于面积的叶片营养元素含量等, 由此与其他叶性状 相互关联所形成的叶经济谱(Wright et al., 2004; Donovan et al., 2011; Díaz et al., 2016), 可量化植物 利用环境资源的“投资-收益”权衡策略(陈莹婷和许 振柱, 2014), 综合反映植物功能性状与环境和生态 系统功能的关系, 对模拟和预测气候变化对生态系 统的影响, 指导生物多样性保护和生态系统管理政 策的制定等具有重要的生态学意义(刘晓娟和马克 平, 2015; 王常顺和汪诗平, 2015; Díaz et al., 2016)。

在野外测定叶面积时, 针对一个样地中的目标 植物, 除了极其特殊的情形, 如无叶或鳞叶植物、针 叶植物等, 通常是每种植物选择 10 株成熟个体, 随 机采摘相对幼嫩但伸展、硬化且无病虫害、无附生 植物的健康叶片 20 个; 至少保证每个物种采集 10 个 叶片, 在叶片稀少时最起码也需要 5 个植株, 每个植 株采摘 2 个叶片, 或者在成熟个体稀少时最少有 4 个 植株、每个植株采摘5个叶片; 保湿、冷藏储存, 并 尽快利用可自动测量的叶面积仪 (如 LI-COR或 Delta-T)或者人工测定的扫描仪进行叶面积测定 (Cornelissen et al., 2003; Pérez-Harguindeguy et al., 2013)。然而, 受系统进化地位所决定的科属分类以 及诸多环境因素的影响, 植物的叶片大小存在显著 的种间变异与较显著的种内变异(Westoby et al., 2002; Albert et al., 2010; Bolnick et al., 2011; Auger
\& Shipley, 2013; Siefert et al., 2015; Messier et al., 2017)。因此, 在同一地点与不同地点, 同一植物生 长于不同生境, 同一植物的不同植株, 甚至同一植 株的不同部位(如阴面、阳面), 叶片大小均会存在差 异。因此在野外实际采样过程中, 比如针对森林中 的优势木本植物, 在理想情况下, 一般会分别选择 不同胸径等级或仅大胸径等级的植株 3-5株, 在每 个植株的向阳部位(冠层)随机采摘5-10个叶片, 或 者在每个植株的四个方位与顶部随机分别摘取3-5 个叶片, 再进行叶面积的扫描测定。但成熟大树一 般较高大, 冠层位置高, 无法有效获取叶片; 而幼 小树木的代表性又不强。

那么, 测定叶面积时需要摘取一定数量的叶片 作为重复, 但叶片大小具有显著的种内与种间差异, 给野外取样带来一个很大的困惑: 对每一个植株, 叶片取样数量多少才算合适? 对一种植物, 采集多 少个植株才能代表一个植物种叶片大小的普遍特 征? 取样数量少, 不一定能完全反映植物叶片性状 特征; 而取样数量多, 则耗费太多的人力和时间。叶 片取样质与量的权衡, 是目前一个需要解决的问题。

因此, 本研究以浙江省金华市金华山的常绿落 叶阔叶混交林中的两种优势树种为对象, 通过设计 多植株、大批量叶片采样，统计分析同一树种内不 同植株在不同生境中的叶面积变异特征，从而回答 选择多少植株, 采集多少叶片, 才能代表一种植物 的叶片大小特征的真实值这个科学问题。本研究可 为今后的叶片性状测定取样数量(最适值)提供证据, 以最优的叶片取样数量、最少的时间与人力成本而 达到最适的测定效果。该研究也可进一步对确定其 他植物器官性状的取样数量具有借鉴意义。

\section{1 材料和方法}

\section{1 研究区概况}

金华山地处中亚热带北缘, 位于浙江省中西部 金华市北15 km处，金倠盆地东部，系龙门山脉的 
支脉，因横亘于金华市北，故俗称北山。整个山体跨 越 $119.48^{\circ}-119.82^{\circ}$ E, 29.15은 $29.27^{\circ} \mathrm{N}$, 海拔通常在 100-1 200 m, 主峰大盘尖海拔1 314 m。研究区位于 金华山南坡双龙洞国家森林公园内, 属亚热带山地 季风气候。据金华气象站 $\left(119.65^{\circ} \mathrm{E}, 29.12^{\circ} \mathrm{N}\right.$, 海拔 $626 \mathrm{~m})$ 1953-2014年的纪录, 该地区年平均气温为 $17.7{ }^{\circ} \mathrm{C}, 1$ 月平均气温 $5.2{ }^{\circ} \mathrm{C}, 7$ 月平均气温 $29.5{ }^{\circ} \mathrm{C}$, 极端低温达-9.6 ${ }^{\circ} \mathrm{C}$, 极端高温为 $41.5{ }^{\circ} \mathrm{C}$; 年降水 量可达1 $436.8 \mathrm{~mm}$, 主要集中在3-9月, 占全年降水 量的 $76.7 \%$, 但以 6 月份梅雨季节的降水量最大, 平 均达 $252.2 \mathrm{~mm}, 3-6$ 月平温期占降水集中期降水量 的70\% (平均月降水量191 mm), 而7-9月高温期降 水反而不是最高(平均月降水量 $112 \mathrm{~mm}$ ); 年日照率 为 $43 \%$, 无霜期 256 天。该地区土壤以亚热带山地黄 壤为主, 植被具有亚热带常绿阔叶林、常绿落叶阔 叶林和灌从等垂直带谱, 但受人为干扰影响大, 是 人为干扰下典型的亚热带常绿润叶林退化植被, 山脚至中坡(200-700 m) 以次生性的常绿阔叶林、 常绿落叶阔叶混交林和针阔混交林、次生灌从为主, 中坡至上坡(600-1000 m)主要为马尾松(Pinus massoniana)人工林、杉木(Cunninghamia lanceolata)林、 柳杉(Cryptomeria fortunei)林、黄山松(Pinus taiwanensis)林和山地灌丛(郭水良等, 1993; 方芳和郭 水良, 2004)。

\section{2 野外采样与测试}

取样点位于 $119.62^{\circ} \mathrm{E} 、 29.20^{\circ} \mathrm{N}$ 附近山谷两侧 的常绿落叶阔叶混交林, 山体较陡峭, 坡度 $20^{\circ}-40^{\circ}$ 。群落乔木层优势树种以木荷(Schima superba)、青冈(Cyclobalanopsis glauca)、枫香树 (Liquidambar formosana) 为主, 其次是苦槠栲 (Castanopsis sclerophylla)、柯(Lithocarpus glaber)、 雉栗(Castanea henryi)等, 少数马尾松大树散生其 间。树高10-15 m, 胸径 10-25 cm, 郁闭度为 0.7 。灌 木层主要由格药柃(Eurya muricata)、南烛(Vaccinium bracteatum)、荚蒾(Viburnum dilatatum)、算盘子 (Glochidion puberum) 等组成, 草本层由䓲 (Pteridium sp.)、鳞毛䕬(Dryopteris sp.)、三脉紫苑 (Aster ageratoides)、芒(Miscanthus sp.)等组成。

选取常绿树种木荷与落叶树种枫香树为研究对 象, 前者取样海拔范围 305-794 m, 后者 457$635 \mathrm{~m}$ 。在2017年7月中下旬, 按照不同的胸径等级: 5-10 cm (D2)、10-15 cm (D3)、15-20 cm (D4)、20-
$30 \mathrm{~cm}$ (D5)和30-45 cm (D6), 对前3个等级各选择20 株、后 2 个等级分别选择 15 和 10 株进行取样(大树个 体较少), 等级D1 (0-5 cm)无植物个体。对每个植株 记录其编号和位置、胸径大小、树高、经纬度、海 拔、坡度和坡向等。同时, 将树木冠层划分为上中 下三部分, 利用高枝剪等采样工具, 在其树冠的底 部, 树冠中部的东、南、西、北方位, 以及树冠上部 开阔区域的顶部，共6个方位，分别采摘成熟、健康、 无病害的叶片 5 片, 每株树合计采集叶片 30 片, 编号 封装在密封袋内, 置于便携式冷藏箱内储存。共采 集叶片 5 087片, 其中木荷2 543片, 枫香树2 544片。 在采集叶片的当天, 利用便携式叶面积仪 (LI-3000C, LI-COR, Lincoln, USA)扫描测量每个叶 片的叶面积。

\section{3 统计分析}

利用SPSS 20.0计算木荷与枫香树 2 个树种、5个 胸径等级、10-20株树木、6个采样方位的叶面积平 均值、标准误差、四分位统计及其95\%置信区间; 基 于独立样本 $t$ 检验计算不同胸径、采样方位叶面积的 差异性, 获取不同树种在不同胸径等级与不同方位 的单个叶面积与其总平均值间的偏离程度, 并区分 未成熟树木(胸径 $<20 \mathrm{~cm}$ )和成熟树木(胸径 $\geqslant 20 \mathrm{~cm}$ ) 进一步分析统计。利用R 3.3软件中的Sample程序进 行所有叶面积样本以及有限制条件的叶面积样本、 有放回的随机抽样, 以比较不同取样数量下叶面积 均值及其置信区间的偏移, 利用 $t$ 检验获取目标样 本的均值和均值的 $95 \%$ 置信区间, 并将目标样本与 总体样本之间进行独立样本 $t$ 检验。如果随机选取的 叶面积与总体叶面积没有显著性差异, 则为最优取 样数量。所有图形均利用Golden Software Grapher 9.1.5软件绘制。

\section{2 结果}

\section{1 叶面积特征}

所有叶片的统计分析表明, 木荷和枫香树叶面 积总体呈正态分布, 木荷叶面积平均为(41.60 \pm 10.88) $\mathrm{cm}^{2}$, 介于16.74-100.80 $\mathrm{cm}^{2}$ 之间(图1A), 而 枫香树平均值为 $(57.65 \pm 19.35) \mathrm{cm}^{2}$, 变化范围为 11.31-129.51 cm $\mathrm{cm}^{2}$ (图1B)。二者叶面积差异较大, 无 论是叶面积还是变幅, 常绿乔木木荷均小于落叶乔 木枫香树。

木荷的叶片大小与胸径等级之间呈现极不显著 

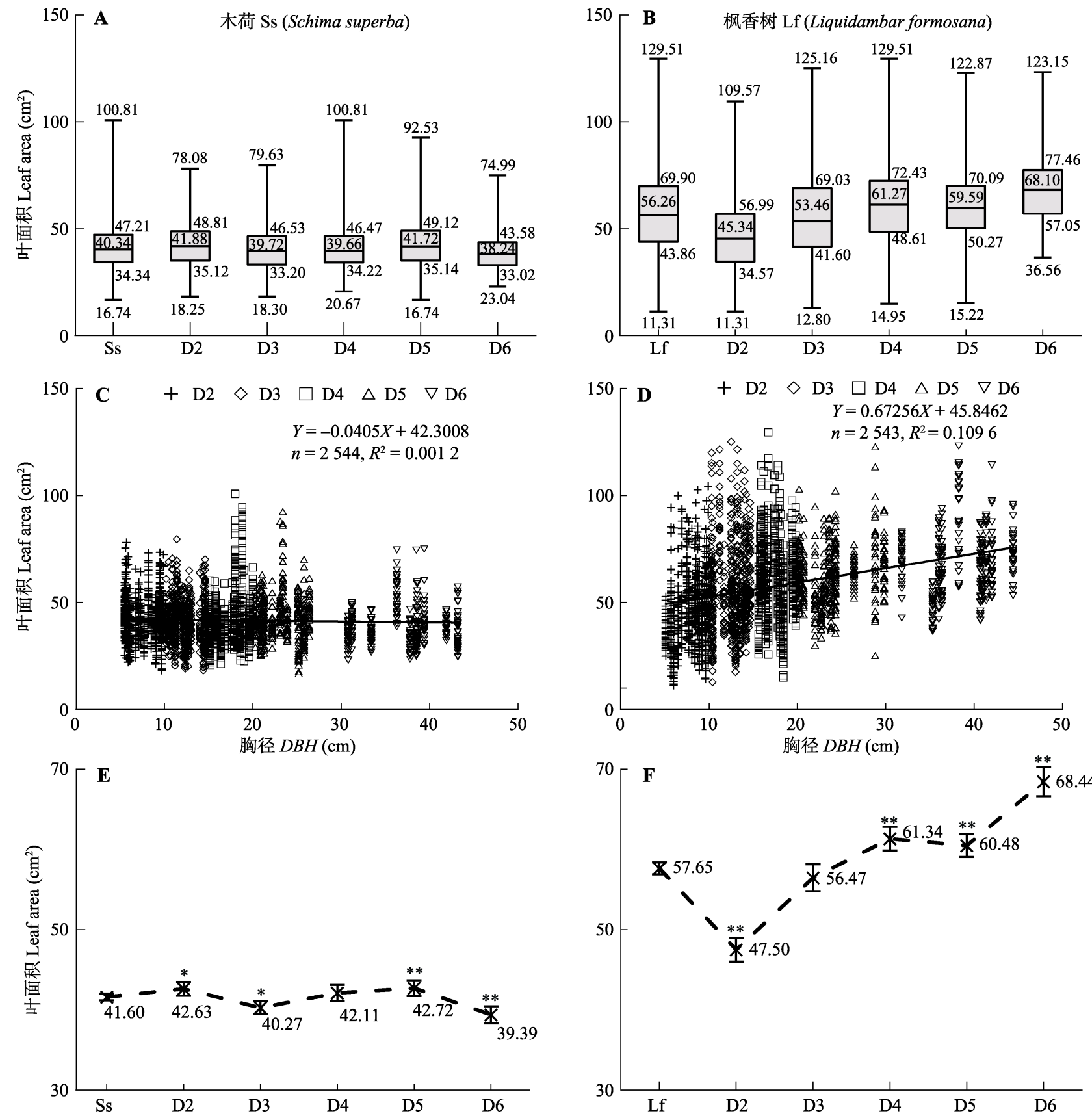

图1 两个树种叶面积与胸径的关系。A, B, 四分位图; C, D, 散点图; E, F, 置信区间图。Ss, Lf, 木荷与枫香树所有胸径等级 样品的平均值。D2-D6, 胸径等级D2-D6。*, $p<0.05 ; * *, p<0.01$ 。

Fig. 1 Relationship between leaf area and diameter at breast height of two tree species. A, B, Quartile map; C, D, Scatter plot; E, F, Confidence interval. Ss, Lf, mean values of all samples in all classes of diameter at breast height for Schima superba and Liquidambar formosana, respectively. D2-D6, D2-D6 classes of diameter at breast height. *, $p<0.05 ;{ }^{* *}, p<0.01$.

的负相关性(图1C), 而枫香树的叶面积却与胸径之 间呈现较显著的正相关性(图1D)。在不同胸径等级 之间，木荷的叶面积差异不显著，仅在D4与D5两个 胸径等级表现出较大的变化区间(图1E); 但枫香树 在不同胸径等级却有较显著的差异(图1D), 在所有 胸径等级均表现出较大的变化区间, 尤其是D2和 D5两个胸径等级。木荷叶面积的平均值和置信区间
在第3和第6胸径等级有轻微异常偏移(图1E), 而枫 香树叶面积则在第 2 和第 6 胸径等级处有较大的异常 位移，胸径越大，置信区间也越向上偏移(图1F), 说 明其叶面积与胸径间的正相关关系越密切。

木荷与枫香树的叶面积与采样方位无相关性 (图2A、2B), 在不同采样方位之间差异也不显著(图 2C、2D), 但木荷在南部方向的叶面积大于其他方位

www.plant-ecology.com 

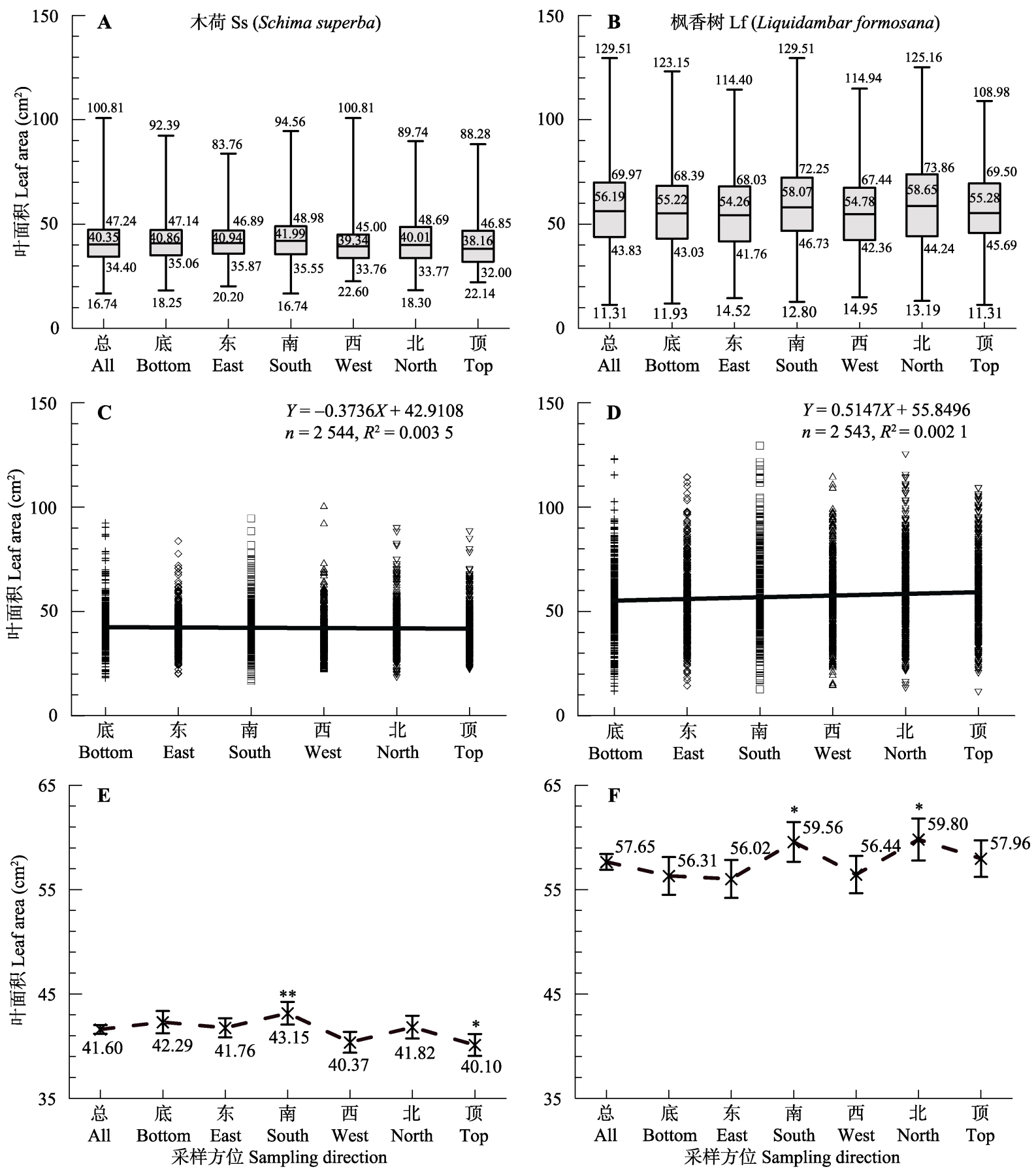

图2 两个树种叶面积与采样方位的关系。A, B, 四分位图; C, D, 散点图; E, F, 置信区间图。*, $p<0.05 ; * *, p<0.01$ 。

Fig. 2 Relationship between leaf area and sampling direction of two tree species. A, B, Quartile map; C, D, Scatter plot; E, F, Confidence interval. *, $p<0.05$; **, $p<0.01$.

的, 顶部和西部的叶面积最小(图2C); 枫香树的叶 面积在南北两个方向均较大, 在东西两个方向较小 (图2D), 且上述所有方位的置信区间均较显著上移 或下移(图2E、2F)。总体来看, 叶面积随采样方位的 偏移(图2E、2F)明显小于随胸径的偏移(图1E、1F)。

\section{2 叶片取样对象与方位}

木荷的大胸径等级(D5-D6)个体的叶面积偏离
均值极显著 $(p<0.01)$, 小胸径等级(D2-D3)显著 $(p<$ $0.05)$, 而中等胸径(D4)则不显著(图3A); 其在南向 和顶部分别偏离极显著与显著, 其他方位则不显著 (图3B)。对枫香树而言, 除胸径等级D3之外, 其他 所有胸径的个体叶面积均极显著偏离均值(图3C); 而其只有南和北两个方位显著偏离均值，其他方位 都不显著(图3D)。 
以成树和非成树单独进行统计检验(图4)发现, 两种树木的成树与非成树在不同采样方位的叶面积 差异不显著(未展示)。木荷由于不同胸径和叶面积 差异较小, 所以成树与非成树的均值与所有树木总
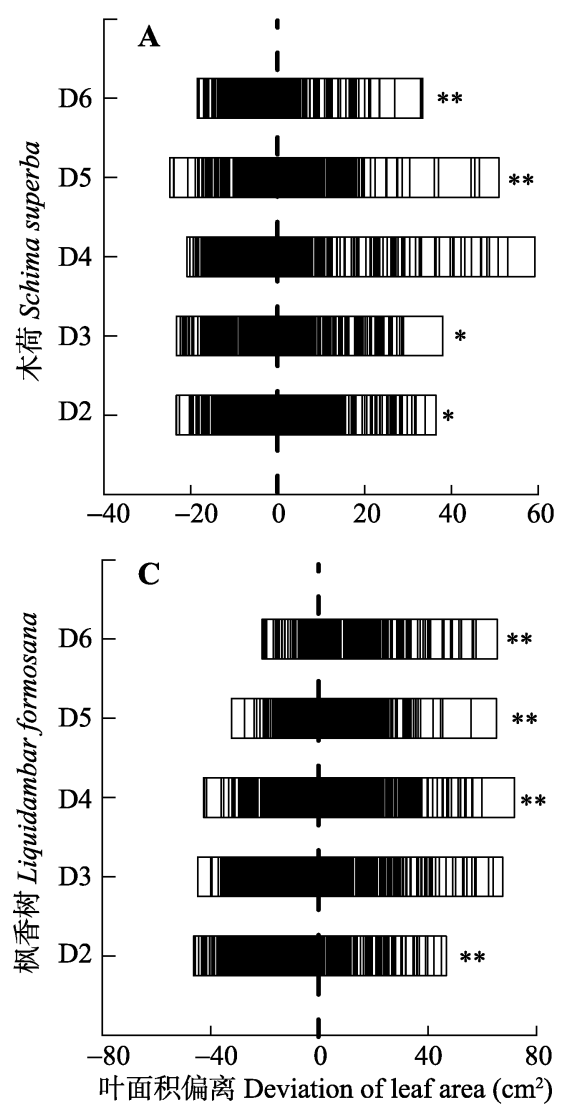

均值之间的差异不大。而枫香树叶面积在成树和非 成树间表现出明显的差异性, 若以成树叶面积均值 代替总叶面积均值则导致总叶面积呈显著的负偏移， 若以非成树叶面积均值代替总叶面积均值则导致叶
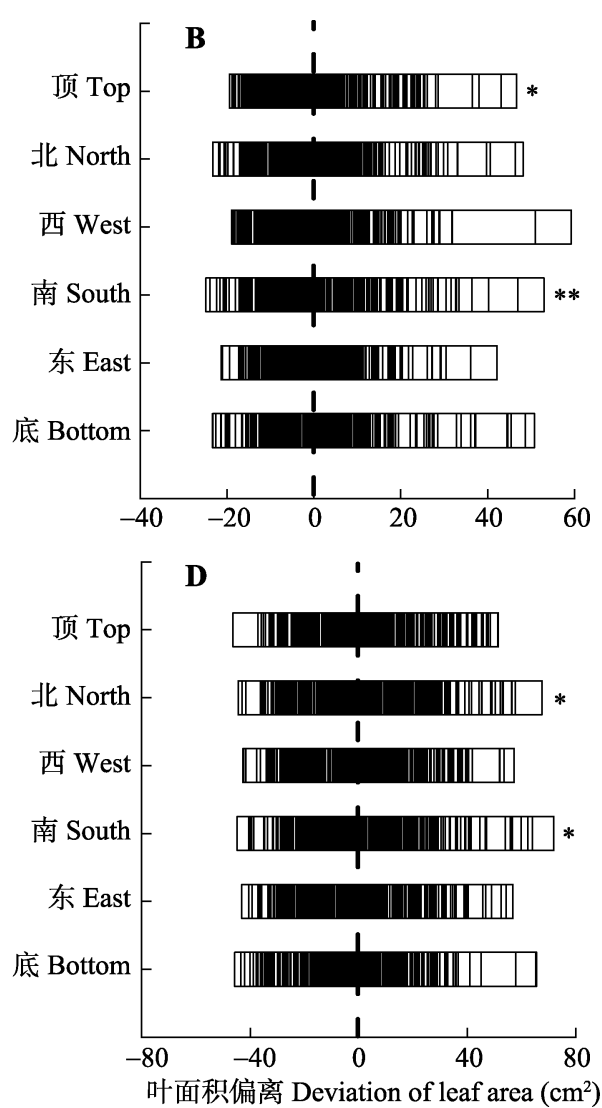

。B, 木荷采样方位。C, 枫香树胸径。D, 枫香树采样方位。

图3 两个树种叶面积在胸径与采样方位的偏离。A, 木荷胸径。 $*, p<0.05 ; * *, p<0.01$ 。坚线表示归零后的均值。

Fig. 3 Deviation of leaf area to diameter and sampling direction of two tree species. A, Diameter of Ss; B, Sampling direction of Ss; C, Diameter of Lf; D, Sampling direction of Lf. *, $p<0.05$; ${ }^{* *}, p<0.01$. The vertical line indicates the mean value returning to zero.

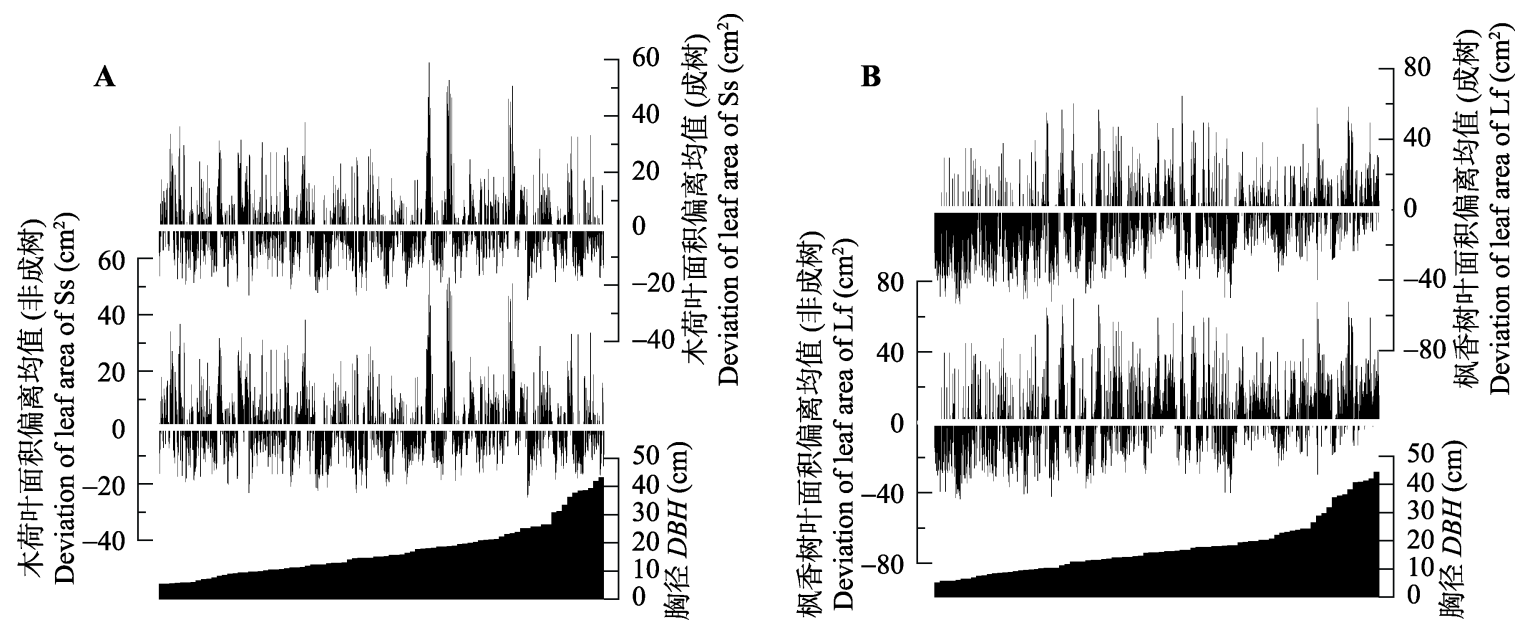

图4 两个树种成树与非成树叶面积在胸径上的偏离。 $\mathbf{A}$, 木荷。 $\mathbf{B}$, 枫香树。横线表示归零后的均值。

Fig. 4 Deviation of mature and non-mature leaf areas to diameter at breast height $(D B H)$ of two tree species. A, Schima superba (Ss). B, Liquidambar formosana (Lf). The horizontal line indicates the mean value returning to zero. 
面积出现整体正偏移。

综合比较可以看出, 中等胸径的植株叶片是最 适的取样材料, 而东西向与底部的叶片是可选的, 但考虑到东方与西方叶片位置较高, 那么选择底部 的叶片也不失一个良策。

\section{3 叶片取样数量}

以所有胸径等级、采样方位的叶片样本为基础 进行的随机抽样与检验表明, 木荷随机抽样大于 734片(图5A), 枫香树大于1 157片(图5C), 所获得的 目标样本与总样本间才无显著性差异。因此, 在不 限定胸径等级和取样方位前提下, 734和1 157分别
是木荷与枫香树叶面积性状研究所需要的最低取样 数量。如果只考虑最佳的取样对象和方位, 即以木 荷 D4胸径和枫香树 D3 胸径等级的底部叶片进行随 机取样, 那么木荷与枫香树叶片的取样数量以 40 (图5B)和170片(图5D)为最适。

\section{3 讨论}

植物的分类地位与环境条件决定其性状特征, 包括叶面积。植物叶面积存在较大的种间变异 (Westoby et al., 2002; Albert et al., 2010; Bolnick et al., 2011; Violle et al., 2012; Siefert et al., 2015;
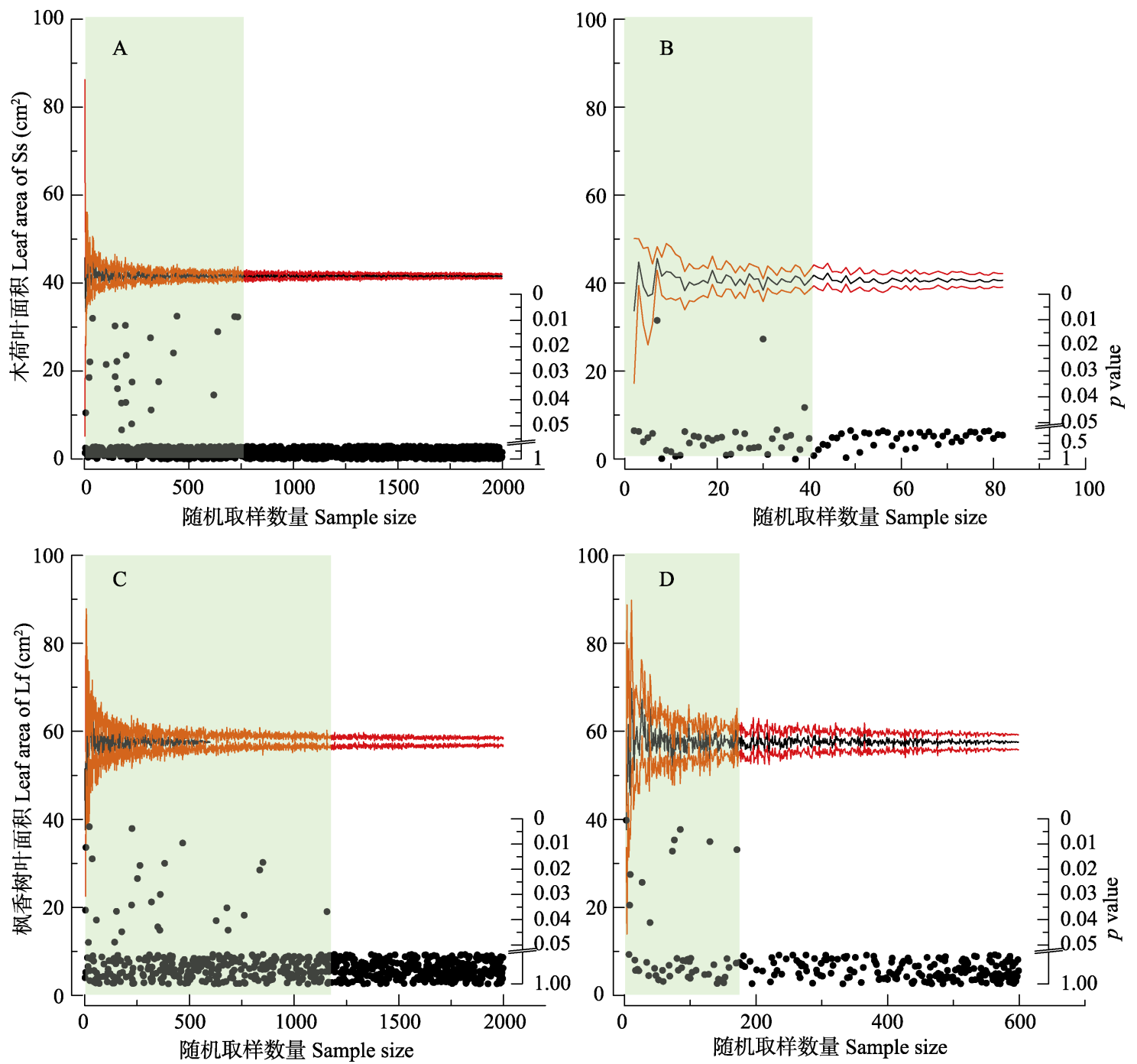

图5 两个树种叶面积的随机抽样均值和 $95 \%$ 置信区间及其差异性检验。 A, C, 全部叶片样品。 B, D, 中等胸径等级、底部叶 片样品。叶面积图上的黑色线代表随机抽样均值, 红色线代表95\%置信区间; 淡蓝色区块表示差异性检验显著区域, 亦即该区 块最右侧的取样数量即为叶片最适取样数量。

Fig. 5 Mean of random sampling, 95\% confidence interval and significant test of leaf areas of two tree species. A, C, All leaf samples. B, D, Leaf samples of medium diameter class and bottom sampling direction. The black line of the leaf area plot indicates the mean value of random sampling, and the red line indicates the 95\% confidence interval; the light blue block is the area of significant difference, indicating that the sample size of the right position of the block is the optimal sampling number of leaves. Ss, Schima superba; Lf, Liquidambar formosana. 
Messier et al., 2017), 因此不同物种叶性状测定时的 叶片取样数量应该不同。而叶片大小的种内变异也 同样不容忽视(Auger \& Shipley, 2013; Bu et al., 2017), 因此在采集同一个物种的叶片时也应该考虑 不同植株的差异。另外，气候、土壤、光照、海拔、 地形等要素也会影响植物叶片的大小(McDonald et al., 2003; Royer et al., 2008; Peppe et al., 2011), 同一 物种的不同植株间和同一个植株内, 叶片大小也可 能存在差异。

因此, 在测定植物叶片大小时, 按照传统和约 定俗成地采摘成熟植株的健康叶片10-20片(Cornelissen et al., 2003; Pérez-Harguindeguy et al., 2013), 在有限的人力、物力和时间条件下，是可以接受的; 但对于植物性状的精细研究来说, 10-20片叶片是远 远不足的, 应该针对不同植物采集不同数量的叶片 开展研究。本研究中木荷的最适采样数量为 40 片叶 片, 枫香树为 170 片叶片, 最适采样数量与树种有 关。木荷为常绿树种, 叶片为较小单叶, 叶面积变异 小, 因此最适的叶片数量较小。而枫香树为落叶树种, 叶片为较大单叶, 叶面积变异大, 因此最适的叶片 数量大于木荷。同时, 常绿树种叶寿命长, 叶面积建 设成本高、消耗大，叶片变异大则不利于其适应环境; 而落叶树种叶寿命短, 建设成本低, 在不同环境、不 同树冠部位, 乃至不同发育阶段采取不同方式适应 环境, 因而其叶片变异比常绿树种大(Aerts, 1995; Givnish, 2002), 这也是木荷叶片最适采样数量小于 枫香树的一个重要原因。

另外, 传统的方法对采样植株的年龄与采样方 位没有具体规定, 通常在野外操作时都是针对大树 向阳的叶片。本研究通过对亚热带常绿落叶阔叶混 交林的一个常绿与一个落叶优势树种的研究发现, 中龄植株树冠底部是较合适的叶片采样对象与方 位。树龄影响叶片大小, 在种间表现出一定的差异, 木荷叶片大小与树龄关系不密切, 而枫香树则较密 切, 二者中龄树的叶片大小与总叶片大小的关系一 致。而取样方位, 亦即光照强度同样影响叶片大小 (李明财等, 2009), 两个树种的叶片大小与取样方位 的关系也与上述树龄关系相同, 但密切程度较小; 通常只是在南、北与顶部的叶片大小变异较大, 东、 西、底部的变异相对较小, 考虑到野外的可操作性, 以底部采样为宜。

上述研究结果可能也适用于其他器官性状测定
的采样, 在今后的植物性状研究中, 无论是叶片还 是茎干、枝条或者根系, 都应该采集较多的植物样本, 并兼顾不同树龄和不同方位。但对不同的植物器官 来说, 到底采集多少数量才是最适的, 仍需要进行 更多植物的观测与实验。同时，也需要更多注意不同 气候带的优势植物的性状; 包括乔木、灌木和草本植 物, 以期发现不同气候带、不同植被类型中, 植物器 官采样数量的差异。

致谢感谢浙江师范大学重点建设项目 (2017XM023和2017PT009)资助。

\section{参考文献}

Aerts R (1995). The advantages of being evergreen. Trends in Ecology and Evolution, 10, 402-407.

Albert CH, Thuiller W, Yoccoz NG, Soudant A, Boucher F, Saccone P, Lavorel S (2010). Intraspecific functional variability: Extent, structure and sources of variation. Journal of Ecology, 98, 604-613.

Auger S, Shipley B (2013). Inter-specific and intra-specific trait variation along short environmental gradients in an old-growth temperate forest. Journal of Vegetation Science, 24, 419-428.

Bolnick DI, Amarasekare P, Araojo MS, Burger R, Levine JM, Novak M, Rudolf VHW, Schreiber SJ, Urban MC, Vasseur DA (2011). Why intraspecific trait variation matters in community ecology. Trends in Ecology and Evolution, 26, 183-192.

Bu WS, Schmid B, Liu XJ, Li Y, Hardtle W, von Oheimb G, Liang Y, Sun ZK, Huang YY, Bruelheide H, Ma KP (2017). Interspecific and intraspecific variation in specific root length drives aboveground biodiversity effects in young experimental forest stands. Journal of Plant Ecology, 10, 158-169.

Chen YT, Xu ZZ (2014). Review on research of leaf economics spectrum. Chinese Journal of Plant Ecology, 38, 1135-1153. [陈芗婷, 许振柱 (2014). 植物叶经济谱的 研究进展. 植物生态学报, 38, 1135-1153.]

Cornelissen JHC, Lavorel S, Garnier E, Diaz S, Buchmann N, Gurvich DE, Reich PB, Ter Steege H, Morgan HD, van der Heijden MGA, Pausas JG, Poorter H (2003). A handbook of protocols for standardized and easy measurement of plant functional traits worldwide. Australian Journal of Botany, 51, 335-380.

Díaz S, Kattge J, Cornelissen JHC, Wright IJ, Lavorel S, Dray S, Reu B, Kleyer M, Wirth C, Prentice IC, Garnier E, Boenisch G, Westoby M, Poorter H, Reich PB, Moles AT, Dickie J, Gillison AN, Zanne AE, Chave J, Wright SJ, Sheremet'ev S, Jactel H, Baraloto C, Cerabolini B, Pierce 
S, Shipley B, Kirkup D, Casanoves F, Joswig J, Günther A, Falczuk V, Rüger N, Mahecha MD, Gorné LD (2016). The global spectrum of plant form and function. Nature, 529, 167-171.

Donovan LA, Maherali H, Caruso CM, Huber H, de Kroon H (2011). The evolution of the worldwide leaf economics spectrum. Trends in Ecology and Evolution, 26, 88-95.

Fang F, Guo SL (2004). DCA ordination on woody plant communities in Beishan Mountain of Jinhua, Zhejiang Province. Journal of Zhejiang Normal University (Natural Science), 27(1), 55-61. [方芳, 郭水良 (2004). 浙江金华 北山木本植物群落的DCA排序. 浙江师范大学学报(自 然科学版), 27(1), 55-61.]

Givnish TJ (2002). Adaptive significance of evergreen vs. deciduous leaves: Solving the triple paradox. Silva Fennica, 36, 703-743.

Guo SL, Liu P, Chen G, Lu X (1993). Observations on the floristic and vegetation in Beishan Mountain of Jinhua in Zhejiang Province. Journal of Zhejiang Normal University (Natural Science), 16(2), 59-67. [郭水良, 刘鹏, 陈刚, 卢晓 (1993). 浙江金华北山植物区系及植被. 浙江师范 大学学报(自然科学版), 16(2), 59-67.]

Li MC, Zhu JJ, Sun YR (2009). Responses of specific leaf area of dominant tree species in Northeast China secondary forests to light intensity. Chinese Journal of Ecology, 28, 1437-1442. [李明财, 朱教君, 孙一荣 (2009). 东北次 生林主要树种比叶面积对光照强度的响应. 生态学杂 志, 28, 1437-1442.]

Liu XJ, Ma KP (2015). Plant functional traits-Concepts, applications and future directions. Scientia Sinica Vitae, 45, 325-339. [刘晓娟, 马克平 (2015). 植物功能性状研究 进展. 中国科学: 生命科学, 45, 325-339.]

McDonald PG, Fonseca CR, Overton J, Westoby M (2003). Leaf-size divergence along rainfall and soil-nutrient gradients: Is the method of size reduction common among clades? Functional Ecology, 17, 50-57.

Meng TT, Ni J, Wang GH (2007). Plant functional traits, environments and ecosystem functioning. Journal of Plant Ecology (Chinese Version), 31, 150-165. [孟婷婷, 倪健, 王国宏 (2007). 植物功能性状与环境和生态系统功能. 植物生态学报, 31, 150-165.]

Messier J, Lechowicz MJ, McGill BJ, Violle C, Enquist BJ (2017). Interspecific integration of trait dimensions at local scales: The plant phenotype as an integrated network. Journal of Ecology, 105, 1775-1790.

Peppe DJ, Royer DL, Cariglino B, Oliver SY, Newman S, Leight E, Enikolopov G, Fernandez-Burgos M, Herrera F, Adams JM, Correa E, Currano ED, Erickson JM, Hinojosa LF, Hoganson JW, Iglesias A, Jaramillo CA, Johnson KR, Jordan GJ, Kraft NJ, Lovelock EC, Lusk CH, Niinemets U, Peñuelas J, Rapson G, Wing SL, Wright IJ (2011). Sensitivity of leaf size and shape to climate: Global patterns and paleoclimatic applications. New Phytologist, 190, 724-739.

Pérez-Harguindeguy N, Diaz S, Garnier E, Lavorel S, Poorter H, Jaureguiberry P, Bret-Harte MS, Cornwell WK, Craine JM, Gurvich DE, Urcelay C, Veneklaas EJ, Reich PB, Poorter L, Wright IJ, Ray P, Enrico L, Pausas JG, de Vos AC, Buchmann N, Funes G, Quétier F, Hodgson JG, Thompson K, Morgan HD, ter Steege H, van der Heijden MG A, Sack L, Blonder B, Poschlod P, Vaieretti MV, Conti G, Staver AC, Aquino S, Cornelissen JHC (2013). New handbook for standardised measurement of plant functional traits worldwide. Australian Journal of Botany, 61, 167-234.

Royer DL, McElwain JC, Adams JM, Wilf P (2008). Sensitivity of leaf size and shape to climate within Acer rubrum and Quercus kelloggii. New Phytologist, 179, 808-817.

Siefert A, Violle C, Chalmandrier L, Albert CH, Taudiere A, Fajardo A, Aarssen LW, Baraloto C, Carlucci MB, Cianciaruso MV, Dantas VD, DeBello F, Duarte LDS, Fonseca CR, Freschet GT, Gaucherand S, Gross N, Hikosaka K, Jackson B, Jung V, Kamiyama C, Katabuchui M, Kembel SW, Kichenin E, Kraft NJB, Lagerstrom A, Le Bagousse-Pinguer Y, Li YZ, Mason N, Messier J, Nakashizuka T, Overton JM, Peltzer DA, Perez-Ramos IM, Pillar VD, Prentice HC, Richardson S, Sasaki T, Schamp BS, Vandewalle M, Wardle DA (2015). A global meta-analysis of the relative extent of intraspecific trait variation in plant communities. Ecology Letters, 18, 1406-1419.

Violle C, Enquist BJ, McGill BJ, Jiang L, Albert CH, Huishof C, Jung V, Messier J (2012). The return of the variance: Intraspecific variability in community ecology. Trends in Ecology and Evolution, 27, 244-252.

Wang CS, Wang SP (2015). A review of research on responses of leaf traits to climate change. Chinese Journal of Plant Ecology, 39, 206-216. [王常顺, 汪诗平 (2015). 植物叶 片性状对气候变化的响应研究进展. 植物生态学报, 39, 206-216.]

Westoby M, Falster DS, Moles AT, Vesk PA, Wright IJ (2002). Plant ecological strategies: Some leading dimensions of variation between species. Annual Review of Ecology and Systematics, 33, 125-159.

Wright IJ, Reich PB, Westoby M, Ackerly DD, Baruch Z, Bongers F, Cavender-Bares J, Chapin T, Cornelissen JHC, Diemer M, Flexas J, Garnier E, Groom PK, Gulias J, Hikosaka K, Lamont BB, Lee T, Lee W, Lusk C, Midgley JJ, Navas ML, Niinemets U, Oleksyn J, Osada N, Poorter H, Poot P, Prior L, Pyankov VI, Roumet C, Thomas SC, Tjoelker MG, Veneklaas EJ, Villar R (2004). The worldwide leaf economics spectrum. Nature, 428, 821-827.

责任编委: 阎恩荣 责任编辑: 李 敏

DOI: 10.17521/cjpe.2018.0087 